\section{Über das Zusammenwirken von Zellfraktionen bei der Glykolyse}

Von Heinz Tiedemann und Jochen Born

Heiligenberg-Institut, Abt. O. MAngoLd, Heiligenberg, Bodensee (Z. Naturforschg. 13 b, 268-269 [1958] ; eingeg. am 24. Januar 1958)

An verschiedenen, aus dem Ehrlich-Ascites-Carcinom gewonnenen Zellfraktionen wurde die Glykolyse untersucht. Dabei zeigte sich, daß der durch hochtouriges Abzentrifugieren aller Strukturbestandteile gewonnene Überstand im Vergleich zu intakten Tumorzellen ${ }^{1}$ aus Glucose nur wenig Milchsäure bildet. Nach Zugabe von Tumormitochondrien zum Überstand steigt die Glykolyse je nach Menge der zugefügten Mitochondrien auf den 2- bis 6-fachen Wert an. Die Mikrosomenfraktion aus Ascites-Tumor steigert in kleiner Konzentration (0,1 g-Äquivalent) die Glykolyse ebenfalls, hemmt aber in höherer Konzentration. Tumormikrosomen verhalten sich also ebenso wie das von $\mathrm{GRAFFI}^{2}$ untersuchte Myeloblastosevirus. Mitochondrien und Mikrosomenfraktion aus Mäuseleber steigern dagegen, in kleiner Menge zum Tumorüberstand hinzugefügt, die Glykolyse nicht oder nur wenig; in größerer Menge zugesetzt, hemmen sie die Milchsäurebildung. In Übereinstimmung hiermit wird in den Ansätzen mit Lebermitochondrien auch weniger Glucose verbraucht (Abb. 1).Der hochtourig zentrifugierte Leberüberstand bildet aus Glucose nur sehr wenig, aus Fructose 1.6-Diphosphat, dagegen größere Mengen Milchsäure.

Schneider und Le $\mathrm{P}_{\mathrm{AGE}}{ }^{3}$ nehmen an, daß die geringe Glykolyse des Überstandes durch Mangel an Adenosindiphosphat (ADP) bedingt ist, da der Überstand allein nur wenig Adenosintriphosphatase (ATP-ase) enthält. Nach Zugabe von Mitochondrien zum Überstand wird durch die in ihnen enthaltene ATP-ase laufend ADP gebildet. - Wir fanden, daß ADP, in einer Konzentration von $6 \cdot 10^{-3} \mathrm{Mol} / l$ zum Tumorüberstand zugesetzt, die Bildung von Milchsäure aus Glucose oder Fructosediphosphat steigert, jedoch nicht in gleichem Maße wie die Tumormitochondrien (Abb. 1).

Da auch Lebermitochondrien und -Mikrosomen aus ATP ADP bilden, müßten sie ebenfalls die Produktion von Milchsäure aus Glucose steigern. Dies ist aber, wie schon erwähnt, nicht der Fall.

Werden an Stelle von Glucose dagegen Glucose-6Phosphat, Fructose-6-Phosphat oder Fructose-1.6-Diphosphat als Substrate verwendet, so wird die Bildung von Milchsäure durch Zugabe von Lebermitochondrien in ähnlicher Weise gesteigert wie durch Zugabe von Tumormitochondrien. - Aktivitätsmessungen der Aldolase und des Fermentsystems Phosphohexoisomerase + Phosphohexokinase nach der kolorimetrischen Methode von Sibley und Lehninger ${ }^{4}$ zeigten, daß die Aktivität dieser Fermente in den beiden Systemen Über-

1 O. WArburg, Über den Stoffwechsel der Tumoren, Berlin 1926; Naturwissenschaften 42, 4011 [1955].

2 A. Graffi, E. J. Schneider u. G. Sydow. Naturwissenschaften 45, 91 [1958].

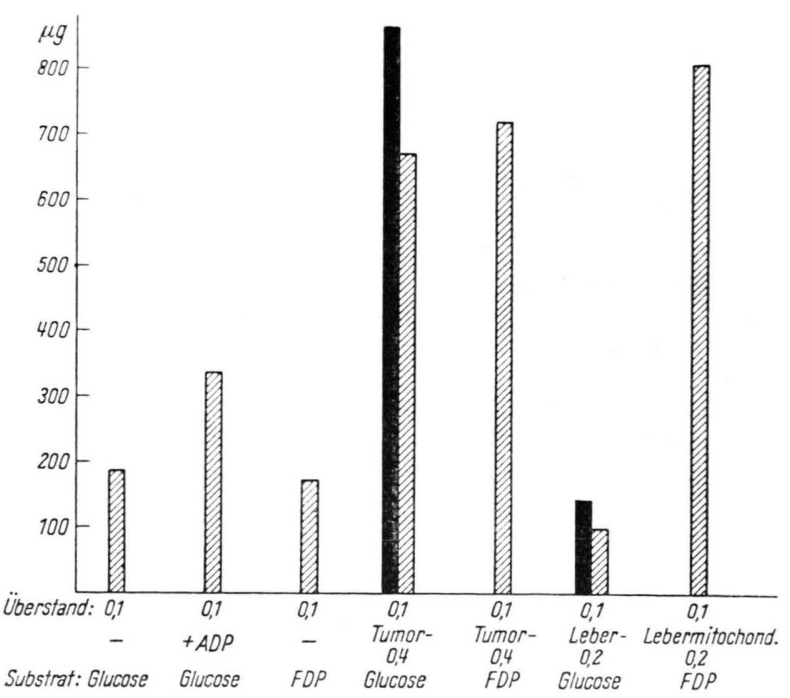

Abb. 1. Glucoseverbrauch und Milchsäurebildung. Die Ansätze $\left(2,2 \mathrm{~cm}^{3}\right)$ enthielten $1 \cdot 10^{-3}-m$. ATP, $1 \cdot 10^{-3}-m$. DPN, $0,5 \cdot 10^{-4}-m$. Nicotinsäureamid, $3 \cdot 10^{-3}-m . \mathrm{MgCl}_{2}, 2 \cdot 10^{-3}-\mathrm{m}$. Phosphat, 2,5 $10^{-2}-m$. KHCO, $1 \cdot 10^{-2}-\mathrm{m}$. Substrat (Konzentrationsangabe in $\mathrm{Mol} / l$ ). Die in den Versuchsansätzen enthaltenen Mengen an Überstand und Mitochondrien sind in Gramm-Äquivalenten angegeben. - $1 \mathrm{~g}$-Äquivalent ist diejenige Menge Überstand oder Mitochondrien, welche bei der Isolierung der Zellfraktionen aus $1 \mathrm{~g}$ intakter Tumoroder Leberzellen erhalten wurde. Die Ansätze wurden durch Zufügen von $\mathrm{KCl}$ isoton gemacht. Versuchsdauer: 30 Min., Temperatur $37^{\circ}$. Gasraum: $5 \% \mathrm{CO}_{2} /$ Luft. - Zur Gewinnung von Mitochondrien und Mikrosomen wurden Lebergewebe oder Ascites-Carzinomzellen mit 0,25-m. Saccharose homogenisiert und aus dem Homogenat die Mitochondrien und Mikrosomen durch fraktioniertes Zentrifugieren isoliert ${ }^{10}$. Die Mitochondrien wurden dann zweimal mit $0,25-m$. Saccharose und zweimal mit $0,16-m$. KCl gewaschen. Zur Gewinnung des Überstandes wurden die Gewebe mit 4 Tln. $0,16-m$. KCl (für Glucosebestimmungen) oder 0,25-m. Saccharose homogenisiert und 60 Min. bei $30000-35000 \mathrm{~g}$ $\left(0^{\circ}\right.$ bis $\left.+4^{\circ}\right)$ zentrifugiert. Milchsäurebestimmung nach Summerson und Barker ${ }^{8}$, Glucosebestimmung nach Nelson ${ }^{9}$. verbrauchte Glucose [III] gebildete Milchsäure.

stand + Lebermitochondrien und Überstand + Tumormitochondrien etwa gleich groß und höher als die der Hexokinase ist.

Die beiden Systeme (Tumorüberstand + Lebermitochondrien und Tumorüberstand + Tumormitochondrien) unterscheiden sich also vor allem durch verschiedene Aktivität des Fermentsystems, welches Glucose-6-Phosphat bildet. Vorläufige Messungen ergaben, daß die Hexokinaseaktivität der Tumormitochondrien wesentlich höher ist als die der Lebermitochondrien. - Die Hemmung der Glykolyse durch Lebermikrosomen und Le-

3 G. A. Le Page u. W. C. Schneider, J. biol. Chemistry 176, 1021 [1948].

4 S. A. Sibley u. A. L. Lehninger, J. biol. Chemistry 177, 859 [1949]. 
bermitochondrien kann z. T. auf die in ihnen enthaltene Glucose-6-phosphatase zurückgeführt werden.

Wie die Aktivitätsunterschiede der Hexokinase, welche bei tierischen Geweben sowohl im Überstand ${ }^{5,6}$ als auch in hochtourig zentrifugierbaren Strukturbestandteilen ${ }^{7}$ enthalten ist, im einzelnen zustande kommen, bedarf noch weiterer Untersuchungen.

* Anm.: Ob bei geringer Substrat-Konzentration, wie sie beim Abbau der Glucose vorliegt, auch die Aktivitäten der Phosphohexoisomerase und der Phosphohexokinase in Gegenwart von Lebermitochondrien verringert sind, bedarf noch weiterer Untersuchungen.

5 S. P. Colowick, G. T. Cori u. M. W. Slein, J. biol. Chemistry 168, 583 [1947].

\section{Messung von Ascorbinsäure auf elektro- chemischem Wege}

\author{
Von K. Damaschke
}

Bundesanstalt für Materialprüfung, Berlin-Dahlem (Z. Naturforschg. 13 b, 269-270 [1958] ; eingeg. am 14. Februar 1958)

Wie schon früher beschrieben ${ }^{1,2,3}$, ist es mit Hilfe der elektrochemischen $\mathrm{H}_{2} \mathrm{O}_{2}$-Meßmethode möglich, nicht nur $\mathrm{H}_{2} \mathrm{O}_{2}$, sondern auch andere reduzierende Stoffe zu messen. WinkeLmann deutete bereits an ${ }^{4}, \mathrm{da} \beta$ man auch Ascorbinsäure auf diesem Wege erfassen kann.

Um einwandfrei messen zu können muß eine lineare Beziehung zwischen dem Gehalt an Ascorbinsäure (A) und dem angezeigten Strom bestehen. Wir gaben daher zu einer 1,2-proz. Phosphatpuffer-Lösung vom $p_{\mathrm{H}} 5,4$ steigende Mengen einer A-Lösung und erhielten so die auf Abb. 1 dargestellte Eichkurve. Da die Zugabe und

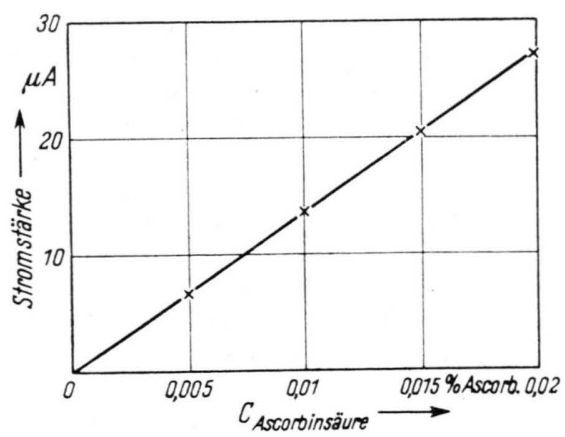

Abb. 1. Eichkurve von Ascorbinsäure in Phosphatpuffer $p_{\mathrm{H}} 5,4$.

Messung in wenigen Sek. auszuführen ist, bestand keine Gefahr, daß sich die zuerst zugegebene A bereits oxydiert hat. Es ist bekannt ${ }^{5}$, daß eine wäßrige Lösung von A bei Zimmertemperatur in 24 Stdn. durch Autoxy-

1 K. Damaschke u. D. Winkelmann, Z. Naturforschg. 11 b, 85 [1956].

2 K. Damaschke, Z. Naturforschg. 12 b, 150 [1957].

3 K. Damaschke, Z. Naturforschg. 12 b, 441 [1957].
Außerdem sind Versuche mit Mikrosomenfraktionen und Mitochondrien aus anderen normalen Geweben sowie mit anderen Tumoren im Gange.

Die Arbeit wurde mit Unterstützung der De u $t s c h$ en Fors chung s. e m e in s c h a f $t$ ausgeführt.

Herrn Prof. Dr. H. L wir für die Úberlassung des Tumormaterials.

${ }^{6}$ M. W. Slein, G. T. Cori u. C. F. Cori, J. biol. Chemistry 186, 763 [1950].

7 R. K. Crane u. A. Sols, J. biol. Chemistry 203, 273 [1953].

8 J. B. Barker u. W. H. Summerson, J. biol. Chemistry 138 , 535 [1941].

9 N. Nelson, J. biol. Chemistry 153, 375 [1944].

10 W. C. Schneider u. G. H. Ноgовоom, J. biol. Chemistry 183. 123 [1950].

dation um $50-60 \%$ absinkt. Spuren von $\mathrm{CuSO}_{4}$ beschleunigen diesen Vorgang, geringe Mengen von $\mathrm{K}_{4}\left[\mathrm{Fe}(\mathrm{CN})_{6}\right]$ oder KCNS stabilisieren die Ascorbinsäure. Da in den meisten Fällen die Oxydation durch Sauerstoff verursacht wird, lag es nahe, auch diesen elektrochemisch mitzumessen. Es konnte auf diese Weise die Oxydationsgeschwindigkeit der A zu Dehydro-A in Abhängigkeit von der $\mathrm{O}_{2}$-Konzentration und der AKonzentration untersucht werden.

In den folgenden Abb. 2 und 3 sind bei der Angabe der $\mathrm{O}_{2}$-Konzentration in $\mathrm{Mol} / l \mathrm{O}_{2}$ halbe Mole gemeint, da $1 / 2 \mathrm{Mol} \mathrm{O}_{2}$ mit $1 \mathrm{Mol} \mathrm{A}$ reagiert.

Es wurde zunächst $\mathrm{zu}$ einer geringen $\mathrm{O}_{2}$-Konzentration $\left(\sim 3 \% \mathrm{O}_{2}\right)$ ein Überschuß an $\mathrm{A}\left(21 \cdot 10^{-4} \mathrm{Mol} / l\right)$ gegeben und die $\mathrm{O}_{2^{-}}$und A-Konzentrationsänderung

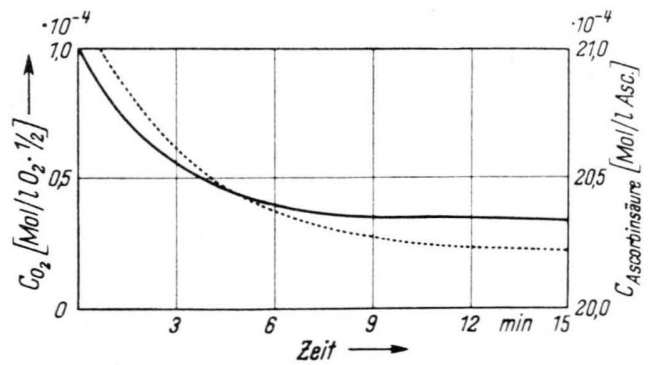

Abb. 2. Abhängigkeit der Oxydationsgeschwindigkeit der Ascorbinsäure, bei einem Überschuß derselben, von der $\mathrm{O}_{2}$ Konzentration.

laufend verfolgt. Wie aus der Abb. 2 zu ersehen ist, wird die Oxydationsgeschwindigkeit der $\mathrm{A}$ in demselben Maße geringer, wie die $\mathrm{O}_{2}$-Konzentration abnimmt. Die Kurven gehen parallel, d. h. die A-Konzentration nimmt durch die Oxydation in demselben Maße ab wie der $\mathrm{O}_{2}$-Gehalt. Beim nächsten Versuch wurde ein $\mathrm{O}_{2}$-Überschuß $\left(\mathrm{O}_{2}\right.$-Sättigung $)$ vorgegeben und eine geringe Menge $\mathrm{A}\left(3 \cdot 10^{-4} \mathrm{Mol} / l\right)$ zugegeben und die

${ }^{4}$ D. Winkelmann, Dissertation, F. U. Berlin 1955.

5 M. Dezelic, J. Grujic-Vasic u. B. Bobarevic, Bull. Soc. Chim. Republ. popul. Bosnie Herzegovine, 2, 55 [1953]. 